\title{
Erratum to: The Controllability of the Gurtin-Pipkin Equation: A Cosine Operator Approach
}

\section{Luciano Pandolfi}

Published online: 20 September 2011

(C) Springer Science+Business Media, LLC 2011

\section{Erratum to: Appl Math Optim (2005) 52: 143-165 DOI 10.1007/s00245-005-0819-0}

Lemma 18 states that $A^{-1}\left[\mathcal{R}_{\infty}\right]^{\perp} \subseteq\left[\mathcal{R}_{\infty}\right]^{\perp}$. Its proof is based on Lemma 17 which is not correct since an integral in the (sketched) computations does not cancel out. A proof of Lemma 18 which does not use Lemma 17 is as follows.

Using formula (7), the Laplace transform of $\theta(t)$ with $\theta(0)=0$ is

$$
\hat{\theta}(\lambda)=-A\left(\frac{\lambda}{\hat{b}(\lambda)} I-A\right)^{-1} D \hat{u}(\lambda) .
$$

Let $u(t)=u_{0} e^{-t}$. For every $\lambda$ (in a right half-plane) and $\xi \perp R_{\infty}$ we have

$$
0=-\langle\xi, \hat{\theta}(\lambda)\rangle=\frac{1}{1+\bar{\lambda}}\left\langle\xi, A\left(\frac{\lambda}{\hat{b}(\lambda)} I-A\right)^{-1} D u_{0}\right\rangle, \quad \forall u_{0} \in U .
$$

The assumptions on $b(t)$ imply that this equality can be extended by continuity to $\lambda=0$ and for $\lambda=0$ we have $\left\langle\xi, D u_{0}\right\rangle=0$ for every $u_{0} \in U$. Hence, if $\xi \perp R_{\infty}$ then $\xi \perp \operatorname{im} D$.

Now we use (1). We use $A=A^{*}$ and we get

$$
-\left\langle A^{-1} \xi, \hat{\theta}(\lambda)\right\rangle=-\left\langle\xi, A^{-1} \hat{\theta}(\lambda)\right\rangle=\left\langle\xi, A\left\{A^{-1}\left(\frac{\lambda}{\hat{b}(\lambda)} I-A\right)^{-1}\right\} D \hat{u}(\lambda)\right\rangle
$$

The online version of the original article can be found under doi:10.1007/s00245-005-0819-0.

L. Pandolfi (凶)

Dipartimento di Matematica, Politecnico di Torino, Corso Duca degli Abruzzi 24, 10129 Torino, Italy e-mail: luciano.pandolfi@polito.it 


$$
=\overline{\left[\frac{\hat{b}(\lambda)}{\lambda}\right]}\left\{\langle\xi, D \hat{u}(\lambda)\rangle+\left\langle\xi, A\left(\frac{\lambda}{\hat{b}(\lambda)} I-A\right)^{-1} D \hat{u}(\lambda)\right\rangle\right\}
$$

When $\xi \perp R_{\infty}$, the first addendum is zero since we proved $\xi \perp \mathrm{im} D$. The second addendum is $\langle\xi, \hat{\theta}(\lambda)\rangle=0$. So, $\left\langle A^{-1} \xi, \hat{\theta}(\lambda)\right\rangle=0$, i.e. $\left\langle A^{-1} \xi, \theta(t)\right\rangle=0$ for every $t$ and every control $u(t)$, as wanted. 